\title{
Publisher Correction: Applications of CRISPR-Cas in agriculture and plant biotechnology
}

Haocheng Zhu, Chao Li and Caixia Gao iD

Nature Reviews Molecular Cell Biology (2020) https://doi.org/10.1038/s41580-020-00288-9 Published online 24 September 2020

Typos in figures were corrected; these changes have been made in the html and PDF versions of the article.

https://doi.org/10.1038/s41580-020-00304-y I Published online 12 October 2020

(c) Springer Nature Limited 2020 